\title{
Meta
}

Journal des traducteurs

Translators' Journal

\section{Ressources documentaires médicales sur Internet : quantité, diversité et qualité}

\section{Aline Francoeur et Marie Brisebois}

Volume 46, numéro 1, mars 2001

Traduction médicale et documentation / Medical translation and documentation

URI : https://id.erudit.org/iderudit/002767ar

DOI : https://doi.org/10.7202/002767ar

Aller au sommaire du numéro

Éditeur(s)

Les Presses de l'Université de Montréal

ISSN

0026-0452 (imprimé)

1492-1421 (numérique)

Découvrir la revue

Citer cet article

Francoeur, A. \& Brisebois, M. (2001). Ressources documentaires médicales sur Internet : quantité, diversité et qualité. Meta, 46(1), 128-144.

https://doi.org/10.7202/002767ar
Résumé de l'article

Internet compte désormais parmi les outils de recherche documentaire du traducteur et, notamment, de celui qui se spécialise dans le domaine médical. Quelles sont les ressources médicales auxquelles il est possible d'accéder sur le Web ? Et surtout, comment trouver de l'information fiable et de qualité dans un réseau caractérisé par un manque flagrant d'organisation et un contenu des plus disparates ? Notre article vise à répondre à ces questions. 


\title{
Ressources documentaires médicales sur Internet: quantité, diversité et qualité
}

\author{
ALINE FRANCEUR \\ Université du Québec à Hull, Hull, Canada \\ MARIE BRISEBOIS \\ Université de Montréal, Montréal, Canada
}

\section{RÉSUMÉ}

Internet compte désormais parmi les outils de recherche documentaire du traducteur et, notamment, de celui qui se spécialise dans le domaine médical. Quelles sont les ressources médicales auxquelles il est possible d'accéder sur le Web? Et surtout, comment trouver de l'information fiable et de qualité dans un réseau caractérisé par un manque flagrant d'organisation et un contenu des plus disparates? Notre article vise à répondre à ces questions.

\section{ABSTRACT}

Over the years, Internet has become a searching tool for the translator who specializes in health sciences, and this situation raises many questions. What kind of documents is it possible to find on the World Wide Web? What is the best way to locate reliable information in a network which content is so disparate and which is mostly characterized by lack of organization? These are some of the questions that will be discussed in this article.

\section{MOTS-CLÉS/KEYWORDS}

traduction médicale, recherche documentaire, Internet, ressources électroniques, qualité de l'information

Pouvoir se documenter vite et bien est une des conditions de l'efficacité d'un traducteur.

Delisle 1997: 85

Le Web foisonne de ressources documentaires en sciences de la santé susceptibles de venir en aide au traducteur qui se spécialise dans le domaine, et cela, à différentes étapes du processus de traduction. Que ce soit pour mieux comprendre le texte de départ, pour résoudre un problème terminologique ou pour se familiariser avec la terminologie du domaine dans la langue d'arrivée, le traducteur trouvera sur Internet des sources d'information variées et de grande qualité. Pour accéder à ces dernières, il devra toutefois connaître quelques principes de base de la recherche documentaire sur Internet. Comme on le sait, dans un réseau informatique aussi vaste, où l'anarchie règne en maître et où la qualité de l'information diffusée est à tout le moins inégale, il n'est pas toujours facile de localiser des ressources fiables pouvant être exploitées à des fins professionnelles.

La recension de ressources électroniques liées à un champ de connaissances aussi vaste que les sciences de la santé est une tâche fastidieuse qui peut difficilement prétendre à l'exhaustivité. Il est en effet pratiquement impossible de répertorier l'ensemble des sites ou des pages Web qui diffusent de l'information en anesthésiologie, cardio-

Meta, XLVI, 1, 2001 
logie, chirurgie, dermatologie, endocrinologie, gynécologie, hématologie, immunologie, neurologie, oncologie, pharmacologie, podiatrie, psychiatrie, rhumatologie... Cependant, et fort heureusement, il existe de nombreux outils pouvant servir de point de départ à la recherche documentaire sur Internet. Ces outils permettront au professionnel langagier qui se spécialise en traduction médicale ou pharmaceutique de repérer de précieuses sources d'information dont il saura tirer profit au quotidien. Dans le présent article, nous passerons en revue les principaux outils qui permettent d'accéder à de l'information factuelle (en français ou en anglais), directement exploitable par le traducteur.

\section{Des ressources électroniques nombreuses et diversifiées pour le traducteur médical}

Le recours à des textes spécialisés fait partie de la démarche documentaire du traducteur, peu importe le secteur dans lequel il exerce. Jean Delisle (1997: 85) observe notamment à ce sujet: «L'effort de compréhension d'un texte peut rendre nécessaire la consultation de diverses sources de documentation. Selon le niveau de technicité du texte et le degré de connaissances du traducteur, la recherche peut se limiter aux encyclopédies générales ou nécessiter la consultation d'encyclopédies spécialisées, d'articles de revues ou de monographies.» Pour le traducteur qui se spécialise dans le domaine médical — qui «de tous les domaines, [...] est peut-être celui qui est le plus difficile à appréhender", estime Amal Jammal (1999: 217) — la consultation de monographies ou d'articles scientifiques fait partie d'une démarche documentaire méthodique et rigoureuse.

Dans le domaine médical, où la compréhension pour qui n’est pas médecin peut paraître une réelle gageure, nous prétendons qu'il est possible de comprendre un sujet à l'aide d'une documentation efficace. [...] Nécessité donc, avant de traduire, de se documenter à fond. Mais par où commencer et quoi chercher? Là, nous proposons au traducteur un itinéraire logique. À la lecture du document à traduire, un document qui, dans la plupart des cas, traitera d'une maladie donnée ou encore d'un médicament proposé pour le traitement d'une maladie, il lui faut tout d'abord déterminer quel est l'appareil, le système, l'organe ou les tissus que la maladie attaque. Il lui faut ensuite se documenter sur la structure de cet appareil ou de cet organe, donc sur son anatomie - ou sur son histologie dans le cas des tissus - , puis sur son fonctionnement, donc sur sa physiologie, ensuite sur la maladie elle-même (son étiologie, son évolution, ses signes et symptômes, etc.) et enfin sur le médicament qui est censé la traiter (la classe à laquelle il appartient, sa composition, son mode d'action, son effet thérapeutique, ses effets indésirables, etc.). Voici donc ce que serait l'ordre séquentiel d'une telle démarche documentaire: anatomie; physiologie; pathologie; pharmacologie.» (Jammal 1999:218)

Jusqu'à récemment, la recherche en bibliothèque était la principale avenue empruntée par le traducteur pour se procurer de la documentation spécialisée. Le réseau Internet offre de nouvelles voies d'accès à l'information, et celles-ci peuvent s'avérer particulièrement rapides et efficaces dans la mesure où l'on fait appel à des outils qui permettent de localiser le type de ressources documentaires recherchées. En moins d'une heure de navigation, on constate qu'il existe un nombre impressionnant de sites et de pages Web consacrés à la médecine et que les ressources qu'on y propose sont des plus diversifiées. Articles scientifiques, manuels, rapports de recherche, guides de pratique clinique, compendiums de produits pharmaceutiques, données 
statistiques, brochures à l'intention des patients, encyclopédies, dictionnaires et lexiques sont au nombre des documents qui, dans le domaine médical, peuvent être consultés sur Internet. Il est également possible d'accéder à de nombreuses bases de données, certaines se spécialisant dans la recension bibliographique (Medline, par exemple), d'autres menant directement à des textes, des images ou des documents multimédias.

La nature du texte de départ et le destinataire du texte d'arrivée sont des facteurs à prendre en considération avant de commencer une recherche documentaire. Il faut notamment s'assurer de consulter des documents qui fournissent une information dont le degré de spécialisation et, partant, le vocabulaire employé, correspondent à celui du texte à traduire. Il faut donc savoir, a priori, que les ressources médicales accessibles sur le Web s'adressent principalement à deux groupes d'usagers: d'une part, les spécialistes du domaine, c'est-à-dire les praticiens, les chercheurs et les étudiants en médecine, de même que, d'autre part, le grand public, d'où la nécessité de distinguer les outils qui permettent d'accéder à de la documentation hautement scientifique de ceux qui conduisent plutôt vers de la documentation à caractère plus général.

\section{Les répertoires spécialisés en sciences de la santé}

Pour localiser de la documentation scientifique de haut niveau, destinée avant tout aux chercheurs, aux médecins ou aux étudiants en médecine, la méthode la plus efficace est la consultation de répertoires spécialisés ${ }^{1}$. Généralement issus de l'initiative de documentalistes œuvrant dans le milieu universitaire ou dans des centres de recherche, les répertoires spécialisés sont en fait des carnets d'adresses de sites Web regroupées à l'intérieur de catégories définies. Leur principal intérêt vient du fait que le contenu des sites qu'ils indexent est évalué en fonction de critères de qualité et de pertinence. Autrement dit, avant que l'adresse d'un site ne soit inscrite dans un répertoire spécialisé, des documentalistes ont pris soin de s'assurer de la crédibilité et de la validité de l'information diffusée sur le site en question.

Les répertoires spécialisés sont le fruit d'un véritable travail documentaire. En effet, on ne se contente pas, dans un tel outil, de fournir l'adresse des sites retenus; on présente en outre une description généralement détaillée de leur contenu. Il est ainsi possible, avant même d'avoir visité un site donné, de savoir qui en est l'auteur et d'avoir une idée relativement précise des ressources auxquelles il donne accès (articles, manuels, rapports de recherche, banques d'images, etc.). L'usager évite ainsi de se rendre sur des sites dont le contenu ne correspond pas à ce qu'il recherche. Dans certains répertoires, on propose de plus une appréciation globale de la qualité du site, tant sur le plan du contenu que sur le plan de la conception et de la présentation.

Il n'existe pas actuellement de catalogue central permettant d'accéder à l'ensemble de l'information médicale diffusée sur Internet. Par contre, on dénombre une multitude de répertoires spécialisés en médecine ou en pharmacologie qui permettent de localiser de la documentation scientifique en langue française et en langue anglaise ${ }^{2}$. Ces répertoires sont le plus souvent localisés sur les sites Web de facultés de médecine, de centres hospitaliers, de bibliothèques universitaires, d'associations ou d'organismes gouvernementaux reconnus dans le domaine. 
Dans les répertoires spécialisés en sciences de la santé, les sites sont habituellement présentés de l'une ou l'autre des façons suivantes. Dans presque tous les cas, on propose une liste qui correspond aux grandes divisions du domaine médical: anatomie, anesthésiologie, cardiologie, chirurgie, dermatologie, gynécologie, hématologie, histologie, immunologie, néphrologie, oncologie, pharmacologie, pédiatrie, rhumatologie, traumatologie, etc. Une fois le domaine de son choix sélectionné, l'usager accède à un inventaire de ressources qui s'y rapportent et qui sont classées soit par catégories (par exemple, bases de données, guides de pratique clinique, manuels ou notes de cours destinés aux étudiants en médecine, périodiques électroniques), soit en fonction du genre de site sur lequel elles se trouvent (sites d'associations, d'organismes gouvernementaux, de facultés de médecine, de bibliothèques médicales, etc.). Dans d'autres cas, l'information est présentée de la façon inverse: on accède d'abord à une liste des différentes catégories de ressources accessibles (bases de données, banques d'images, journaux et périodiques, etc.), liste qui conduit ensuite à un index des sites répertoriés classés par domaines de spécialisation. Enfin, même si le principe de base du répertoire demeure le classement thématique des ressources indexées, certains répertoires spécialisés sont néanmoins munis d'un moteur de recherche qui permet la recherche par mots-clés. Lorsqu'une telle option est offerte, il s'agit généralement du moyen le plus rapide pour accéder à l'information, en autant que les options de recherche proposées et la syntaxe d'interrogation à respecter soient connues de l'usager.

\section{Quelques exemples de répertoires médicaux}

Le lecteur trouvera en annexe une liste d'adresses de répertoires spécialisés ainsi que d'autres ressources dont il sera fait mention dans le présent article. Nous avons toutefois tenu à présenter plus en détail certains répertoires dont le contenu est particulièrement riche ou dont les modes de consultation ou les regroupements thématiques proposés sont les plus raffinés.

Le Catalogue et index des sites médicaux francophones (CISMeF) (<http://www. chu-rouen.fr/cismef/>), initiative du Centre hospitalier universitaire de Rouen, constitue actuellement l'outil le plus efficace pour repérer des ressources électroniques de langue française. Différents modes de consultation sont proposés: entre autres, un index alphabétique de mots-clés (qui correspondent pour la plupart à des pathologies), un index thématique des grands domaines de spécialisation liés à la pratique médicale, une liste de ressources regroupées par catégories, et un moteur de recherche permettant d'effectuer des recherches par mots-clés. Mentionnons en outre que les sites indexés dans le CISMeF font l'objet d'une description très détaillée. Un autre répertoire particulièrement bien conçu permet la localisation de ressources fiables en langue française. Il s'agit du répertoire Promedical.net (<http://www.promedical.net/ $>)$, conçu par des praticiens, et dont le contenu est soumis à l'appréciation d'un comité scientifique formé d'une cinquantaine de spécialistes des différents domaines représentés. Les ressources proposées sont regroupées en quatre grandes catégories: disciplines médicales (par exemple, allergologie, cardiologie, chirurgie, immunologie, etc.), secteur paramédical (par exemple, infirmerie, kinésithérapie, orthophonie, etc.), médecines alternatives (par exemple, acupuncture, aromathérapie, homéopathie, ostéopathie, etc.) et autres thèmes (par exemple, formation médicale, industrie 
pharmaceutique, médecine vétérinaire, santé publique, etc.). En plus d'une description détaillée du contenu des sites répertoriés, on attribue une appréciation globale qui rend compte à la fois du contenu et du contenant (ergonomie et conception du site). Les sites sont en outre classés par pertinence: on signale d'abord les incontournables, puis les autres sites à visiter. La Bibliothèque médicale A. F. Lemanissier $(<$ http://www.bmlweb.org $>)$, dont l'objectif est de repérer et de diffuser l'information médicale destinée aux médecins, pharmaciens, biologistes et étudiants en médecine, propose aussi sur son site un répertoire intéressant, muni de deux moteurs de recherche, l'un permettant d'effectuer des recherches à l'intérieur du site même, l'autre permettant de lancer une recherche plus générale sur le Web. Le site fournit un accès à Medline et diffuse une série de dossiers thématiques constitués à partir de ressources textuelles électroniques. Toutefois, ce répertoire est surtout intéressant pour localiser des articles scientifiques ou des images; il dresse en effet une liste très complète de périodiques médicaux (accessible par le lien «Périodiques») ainsi que de banques d'images (accessibles par le lien «Images»).

Pour localiser des ressources en langue anglaise, on pourra consulter le répertoire Medical Matrix (<http://www.medmatrix.org/reg/login.asp $>$ ), qui compte sans doute parmi les plus complets à l'heure actuelle. Conçu par un comité scientifique formé de membres d'un groupe de travail qui porte le nom d'American Medical Informatics Association's Internet Working Group, Medical Matrix offre différentes options de recherche: entre autres, la recherche par mots-clés, la consultation d'un index de domaines de spécialité et la consultation d'un index de pathologies. Il est nécessaire de s'inscrire pour consulter le répertoire; cependant, l'inscription est gratuite. On propose une appréciation des sites recensés, en plus d'une description détaillée de leur contenu. Si l'on cherche surtout des ressources en pharmacologie, on pourra se tourner vers la bibliothèque virtuelle du College of Pharmacy de l'Université de l'Oklahoma (<http://www.cpb.uokhsc.edu/pharmacy $>$ ). Cette bibliothèque offre de nombreux liens vers des associations du domaine, des entreprises pharmaceutiques, des revues spécialisées, des groupes de discussions et des listes de diffusion. La recherche dans le répertoire peut s'effectuer directement à partir du menu proposé ou à l'aide d'un moteur de recherche interne. La longue liste de signets établie par le College of Pharmacy de l'Université Dalhousie $(<\mathrm{http}: / /$ www.dal.ca/ pharmwww/links $>$ ) constitue également un point de départ intéressant pour localiser des ressources de langue anglaise en pharmacologie. Les sites indexés sont classés par catégories: la catégorie "Government Health Organizations», qui recense des sites gouvernementaux canadiens et américains, est particulièrement riche, de même que la catégorie «Pharmacy and Other Associations », qui signale un grand nombre de sites d'associations canadiennes et internationales du domaine.

La plupart des bibliothèques d'universités qui offrent un cursus médical colligent des index de ressources électroniques dans le domaine. Du côté des universités canadiennes, signalons le site Web de la Bibliothèque des sciences de la santé de l'Université de Montréal (<http://www.bib.umontreal.ca/SA $>$ ), qui répertorie un nombre important de ressources de langue française et de langue anglaise accessibles par différents liens hypertextes. Ainsi, le lien « Ressources d'Internet en santé, triées par sujet» conduit à une liste de domaines de spécialité, chacun des noms de domaine pointant vers des ressources regroupées par catégories. Le lien «Manuels électroniques» mène à une liste de manuels classés par ordre alphabétique de titre et par domaine de 
spécialité. Il est par ailleurs possible, en activant le lien «Sites généraux en sciences de la santé", de consulter un répertoire de sites d'associations, d'organismes, d'institutions et de groupes de recherche québécois, canadiens et internationaux. Une liste de périodiques électroniques, un répertoire de guides de pratique clinique de même qu'une liste de sites utiles pour obtenir des statistiques dans le domaine des sciences de la santé comptent aussi parmi les ressources auxquelles le site permet d'accéder. Du côté des universités européennes, signalons le répertoire constitué par la Bibliothèque centrale de médecine de l'Université libre de Bruxelles $(<\mathrm{http} / / / \mathrm{www} . b i b$. ulb.ac.be/Bcm $>$ ), qui représente un vaste inventaire de sites classés par champs de spécialisation. Les sites référencés font l'objet d'une description détaillée. Les universités américaines fournissent aussi des outils de recherche intéressants dans le domaine médical. Mentionnons, entre autres, le site de la Hardin Library for the Health Sciences (Université de l'Iowa) (<http://www.lib.uiowa.edu/hardin/md $>$ ), métarépertoire qui recense essentiellement des répertoires spécialisés en sciences de la santé. De même, le catalogue virtuel Biosites, conçu par le National Network of Libraries of Medicine $(<$ http://www.library.ucsf.edu/biosites $>)$, qui se distingue par la qualité des fiches descriptives fournies pour chacun des sites répertoriés. Différents modes de consultation du catalogue sont de plus proposés: on peut effectuer une recherche par motsclés, naviguer dans l'index des titres des documents répertoriés ou encore naviguer dans l'index des domaines représentés. L'Université Harvard propose aussi sur le site de sa bibliothèque de médecine ( $<$ http://countweb. med.harvard.edu $>$ ) un catalogue utile pour repérer des bases de données en ligne, des périodiques électroniques et diverses autres ressources regroupées par domaines de spécialisation. Le contenu des sites répertoriés n'est pas décrit en tant que tel, mais cette lacune est compensée par les regroupements précis qui sont proposés. Finalement, mentionnons un répertoire d'origine coréenne, le répertoire MedMark ( $<\mathrm{http}: / / \mathrm{www}$.medmark.org $>$ ), élaboré par les documentalistes de l'École de médecine de l'Université nationale de Chungbuk. On peut y effectuer des recherches par mots-clés ou consulter un index thématique. Le classement des différentes sources d'information est clair et le site donne accès à un très grand nombre de ressources, pour la plupart de langue anglaise.

\section{Les répertoires spécialisés en sciences (tous les domaines scientifiques)}

En plus des nombreux répertoires spécialisés en sciences de la santé, il existe des répertoires à contenu plus général, qui recensent des ressources électroniques dans tous les domaines scientifiques, qu'il s'agisse des sciences pures ou des sciences humaines. Tout comme les répertoires spécialisés en médecine, ces outils sont généralement conçus par des documentalistes professionnels et proposent une information destinée principalement aux chercheurs et aux spécialistes des différents champs de connaissances représentés. Les sites indexés font l'objet d'une description détaillée et leur contenu est soumis à une évaluation préalable. La plupart des répertoires scientifiques existants sont d'origine américaine et renvoient principalement à de la documentation en anglais. 


\section{Quelques exemples de répertoires spécialisés en sciences}

Parmi les répertoires spécialisés dans les domaines scientifiques, mentionnons Infomine (<http://infomine.ucr.edu/search/bioagsearch.phtml $>$ ), bibliothèque scientifique virtuelle particulièrement intéressante de par les différents modes de consultation offerts: recherche par mots-clés, navigation dans un index de thèmes généraux, navigation dans un index de mots-clés, navigation dans un index de titres de sites, et navigation dans une table des matières qui contient à la fois les thèmes traités et les titres des sites répertoriés. Le seul inconvénient de cet outil vient du fait que les ressources en sciences de la santé sont regroupées avec les ressources en biologie et en agriculture. Par conséquent, les index proposés contiennent des liens qui ne sont pas nécessairement pertinents lorsqu'on se spécialise essentiellement dans le domaine médical. Une façon efficace de contourner cet inconvénient est d'utiliser le moteur de recherche interne et de lancer la recherche à partir de mots-clés précis, qui cernent bien le sujet sur lequel on souhaite se documenter.

Le répertoire Academic Info contient pour sa part une section «Health and Medicine» (<http://www.academicinfo.net/med.html $>)$ constituée d'une série de liens vers des ressources diverses: répertoires spécialisés dans le domaine médical, sources d'information pour les consommateurs, ressources dictionnairiques, bases de données, organismes, etc.

Le répertoire Argus Clearinghouse (<http://www.clearinghouse.net $>$ ) est en fait un répertoire de répertoires scientifiques; il peut donc mener à des répertoires médicaux, dont des répertoires spécialisés dans des secteurs précis de la médecine (oncologie, anatomie, etc.). L'usager a le choix du mode de consultation; il peut soit naviguer dans l'index proposé, soit interroger le moteur de recherche interne.

L'un des rares répertoires scientifiques qui conduit à de la documentation française est Sapristi (Sentiers d'accès et pistes de recherche d'informations scientifiques et techniques sur l'Internet: <http://csidoc.insa-lyon.fr/sapristi/digest. html>), conçu par l'Institut national des sciences appliquées de Lyon. Les sites indexés par Sapristi sont regroupés en deux grands thèmes: par catégorie de documents (banques de données, brevets, cours, dictionnaires, rapports techniques et scientifiques, etc.) et par catégories de sites (sites de laboratoires de recherche, d'organisations nationales et internationales, de sociétés, etc.). Seuls certains sites répertoriés font l'objet d'une description.

\section{Les portails médicaux}

Les portails, apparus depuis peu sur le Web, se caractérisent par le nombre de ressources et de services proposés à partir de leur page d'accueil: service de courrier électronique, groupes de discussion, grands titres de l'actualité, petites annonces, boutiques en ligne, etc. Le principe du portail connait un franc succès dans le domaine médical. Les portails médicaux sont aussi nombreux, voire plus nombreux, que les répertoires spécialisés dans le domaine. Toutefois, il est important de savoir que l'information diffusée par l'entremise de ces deux catégories d'outils est fondamentalement différente. D’une part, les répertoires tels que le CISMeF et Medical Matrix, tout comme les listes de signets diffusées sur les sites institutionnels, donnent accès à des ressources qui, dans la majorité des cas, sont hautement spécialisées. D'autre part, les portails médicaux tels que Achoo ( $<$ http://www.achoo.com $>$ ), 
Atmedica $(<$ http://www.atmedica.com $>)$, Medisite $(<$ http://www.medisite.fr $>)$ et Medscape $(<$ http://www.medscape.com $>$ ) ont un contenu plus léger, qui s'adresse avant tout au grand public. Les thèmes abordés dans les pages d'accueil des portails médicaux ont souvent trait, par exemple, à la beauté (bronzage, cosmétologie, régimes amaigrissants, etc.), à la mise en forme, à la nutrition ou encore à la sexualité, et sont généralement regroupés selon qu'ils concernent les enfants, les personnes âgées, les hommes ou les femmes.

Les portails constituent des outils efficaces pour consulter de courts textes journalistiques à caractère médical, des brochures destinées aux patients ou des fiches sommaires de symptômes associés à diverses pathologies. Certains portails proposent également à l'usager des dossiers thématiques qui ont trait à différents problèmes de santé, dossiers qui renvoient à des textes de vulgarisation rédigés en langue courante. Les portails se caractérisent enfin par une interface lourde de bannières publicitaires et d'animations qui distraient l'usager davantage qu'elles ne le renseignent.

Il importe par ailleurs de mentionner que la qualité de l'information diffusée par l'intermédiaire des portails médicaux a parfois été mise en doute. Philippe Éveillard (2000: 327) soutient notamment que les portails médicaux «lèvent des fonds, s'affichent dans les gazettes, mais ne produisent que du vent». Selon lui, «l'information délivrée par les institutions est de bien meilleure qualité que celle distribuée par les portails». On peut tirer de ce commentaire la conclusion suivante: il faut exercer un jugement particulièrement critique vis-à-vis de l'information diffusée sur les portails, surtout si l'on compte exploiter cette information dans un but professionnel.

\section{Quelques exemples de portails médicaux}

D’après Philippe Éveillard (2000: 327), Atmedica (<http://www.atmedica.com>) « est la figure la plus emblématique des portails médicaux de nouvelle génération ». Il s'agit d'un portail exclusivement francophone (sur le plan de la présentation et du contenu) et dont la page d'accueil propose de nombreuses options à l'usager, notamment un moteur de recherche interne. L'onglet «Thèmes» renvoie à une liste de domaines de spécialisation: diabétologie, économie de la santé, génétique, infectiologie, etc. Une fois le domaine sélectionné, on accède à une liste plutôt restreinte de ressources, ressources qui se limitent dans la plupart des cas à de courts articles, à des liens vers des forums de discussion ou à de l'information relative aux congrès et à la formation dans le domaine. L'onglet «Rubriques» conduit pour sa part à un menu bien garni, dont les options ne sont cependant pas toutes pertinentes pour le traducteur. Il est notamment possible de consulter à partir de ce menu les dossiers d'Atmedica (brefs dossiers sur différentes pathologies), un dictionnaire médical français/anglais, une banque d'images, une revue de presse internationale, des petites annonces (offres d'emplois, location de locaux professionnels, demandes d'emploi, etc.), de l'information sur les certificats médicaux et, étrangement, un dictionnaire informatique ainsi que des chroniques d'actualité dans le domaine de l'informatique. Enfin, un menu permanent présenté en haut de l'écran permet d'accéder à des listes de diffusion, des annonces, des annuaires de personnes qui œuvrent dans le domaine médical, une boutique micro, une boutique voyages, etc. Compte tenu de la somme 
considérable d'information présentée dans la page d'accueil du site, la consultation n'est pas nécessairement facile ni agréable.

Caducée $(<\mathrm{http} / / / w w w . c a d u c e e . n e t>)$, comme la plupart des portails médicaux, est muni d'un moteur de recherche interne et renvoie à différentes catégories de documents: informations (revue de presse, nouvelles régionales, émissions santé), annuaires (dont un intéressant annuaire de sites) et services à la communauté (service de courrier électronique, forums de discussion, listes de diffusion, petites annonces). Parmi les ressources textuelles qu'on y trouve, mentionnons les fiches techniques, qui traitent de façon concise de sujets liés notamment à la diététique, ainsi que les dossiers santé, classés par champs de spécialisation. Somme toute, Caducée propose des ressources vulgarisées intéressantes et la navigation dans le site est nettement plus simple que dans d'autres outils du même genre.

Le portail Health A to Z $(<\mathrm{http}: / / w w w . h e a l t h a t o z . c o m>)$ constitue un outil intéressant pour repérer de la documentation vulgarisée en anglais. En lançant une recherche par mot-clé, on accède à une liste qui comporte, d'une part, des documents rédigés par les responsables du site et, d'autre part, des ressources externes, c'est-à-dire des liens vers des pages ou sites Web qui traitent du sujet en question. Le contenu des ressources externes répertoriées fait l'objet d'une description détaillée et d'une note appréciative.

\section{Les répertoires et moteurs de recherche généraux}

D'autres outils permettent l'accès à de la documentation dans le domaine des sciences de la santé. On peut notamment penser aux répertoires de sites généraux tels que Yahoo (<http://www.yahoo.com $>)$, La Toile du Québec (<http://www.toile.qc.ca $>)$ et Nomade (<http://www.nomade.fr $>)$. Tous comptent une catégorie «Médecine» ou «Santé» dans laquelle sont indexés des sites dont la consultation peut s'avérer utile au traducteur médical. Toutefois, il faut garder à l'esprit que les répertoires généraux n'ont pas la prétention d'offrir un contenu scientifique et que les sites qu'ils recensent visent en premier lieu le grand public. Les répertoires généraux constituent néanmoins de bonnes pistes pour localiser les sites d'organismes gouvernementaux ou autres.

De même, les moteurs de recherche généraux tels qu'AltaVista $(<\mathrm{http}: / /$ www. altavista.com $>)$, Google $(<$ http://www.google.com $>)$ et Northern Light $(<\mathrm{http}: / /$ www.northernlight.com $>$ ) permettent d'accéder à des ressources documentaires dans le domaine médical. La prudence et la vigilance sont cependant de mise lorsqu'on recourt à cette catégorie d'outils. Il faut se rappeler que, dans les moteurs de recherche, l'indexation des documents est effectuée de façon automatisée, ce qui signifie que le contenu des sites répertoriés n'est pas évalué et que la qualité de l'information peut être très inégale d'un site à l'autre. De plus, certains préalables sont nécessaires à l'utilisation efficace des moteurs de recherche. Il faut, entre autres, connaitre les principes de base de la recherche par mots-clés, comprendre le fonctionnement des moteurs de recherche et être au fait que les options de recherche proposées à l'usager diffèrent d'un moteur à l'autre. La lecture des fichiers d'aide, qui sont généralement accessibles par un hyperlien à partir de la page d'accueil du moteur, permettra de savoir quels sont les opérateurs de recherche qu'il est possible d'utiliser (opérateurs booléens, opérateurs de proximité, troncature, etc.) et de connaître la 
syntaxe d'interrogation particulière à l'outil sélectionné. Enfin, la plupart des moteurs les plus performants proposent deux options de recherche, soit la recherche en mode simple et la recherche en mode avancé. Lorsque cette dernière option est possible, il est préférable de l'utiliser systématiquement puisqu'elle permet généralement de formuler des requêtes plus précises.

\section{Les sites Web d'associations, d'organismes et d'institutions}

Les sites Web des associations, institutions, organismes et sociétés du secteur de la santé représentent également des sources d'information fiables pour le traducteur. On peut penser, entre autres, aux sites de grands organismes tels que l'Organisation mondiale de la santé $(<\mathrm{http}: / /$ www.who.int $>)$, la Food and Drug Administration $(<$ http://www.fda.gov/cder $>)$, le National Institute of Health $(<\mathrm{http} / / /$ www.nih.gov $>)$, l'Institut Pasteur (<http://www.pasteur.fr $>)$ et Santé Canada $(<\mathrm{http}: / /$ www.hc-sc. gc.ca/francais $>$ ). Mais on peut également penser aux sites d'organismes de moindre envergure ou moins connus, qui ne sont pas pour autant négligeables. Citons, à titre d'exemples, le Centre de coopération internationale en santé et développement $(<\mathrm{http} / / /$ www.ccisd.org $>$ ), dont le site Web est multilingue (français, anglais, espagnol et portugais), Enfant et famille Canada/Child \& Family Canada $(<$ http://www.cfcefc.ca $>)$, le Centre canadien de lutte contre l'alcoolisme et les toxicomanies $(<\mathrm{http} / / /$ www.ccsa.ca/cclat.htm >), l'Institut national de la santé et de la recherche médicale $(<\mathrm{http}: / /$ www.inserm.fr/servcom/servcom.nsf $>$ ).

Le contenu des sites Web institutionnels varie grandement, mais certaines rubriques s'y retrouvent presque systématiquement: la présentation de l'organisme et de sa mission, la liste des publications produites, une banque de signets. On trouvera à l'occasion sur ces sites des rapports de recherche, des guides ou brochures destinés aux consommateurs (par exemple, le Guide alimentaire canadien, que l'on peut consulter sur le site de Santé Canada, une brochure sur les transfusions sanguines intitulée Blood Transfusions. Knowing Your Options, sur le site de l'American Medical Association), ou encore les textes de règlements ou de politiques en matière de santé (par exemple, la Loi canadienne sur la santé, sur le site de Santé Canada, la Politique publique de lutte contre les drogues, sur le site de la Mission interministérielle de lutte contre les drogues et la toxicomanie, organisme français). De plus, les sites d'organismes internationaux sont souvent bilingues ou multilingues, ce qui n'est pas sans intérêt pour le traducteur.

Le moyen le plus efficace de localiser les sites Web d'organismes, d'associations ou de sociétés demeure la consultation de répertoires spécialisés. Les listes d'institutions établies par le CHU de Rouen (<http://www.chu-rouen.fr/ssf/insfr.html $>$ ), par la Société de services et de conseils en informatique médicale et télématique $(<\mathrm{http}: / /$ medintel.net/fr/index $2 . \mathrm{htm}>)$ et par la Bibliothèque médicale Lemanissier $(<\mathrm{http} / / /$ www.cybercable.tm.fr/ biblioa/association.html $>$ ) sont des recensions particulièrement riches de sites institutionnels des pays de la francophonie. Pour localiser les sites Web d'institutions américaines, le répertoire Medline Plus (<http://www.nlm. nih.gov/medlineplus/organizations.html $>$ ) est sans contredit une ressource utile, comme d'ailleurs la liste de signets établie par l'Université McGill $(<\mathrm{http}: / / \mathrm{www}$. health.library.mcgill.ca/resource/associat.htm $>$ ), qui renvoie à des associations américaines et canadiennes. 


\section{Les ressources terminologiques en ligne}

Le traducteur médical pourra consulter de nombreuses ressources terminologiques sur Internet: dictionnaires bilingues et monolingues, encyclopédies, glossaires, lexiques et vocabulaires. Les ressources dictionnairiques du domaine médical étant

dispersées sur les millions de sites Web existants, il est pratiquement impossible d'en établir une liste complète. On peut cependant visiter certains sites stratégiques sur lesquels il est possible de localiser des ressources terminologiques. Ainsi, il n'est pas rare que les associations et les organismes du secteur de la santé constituent leur propre glossaire et le rendent disponible sur leur site Web. Par exemple, l'Association d'aide à la recherche cancérologique de Saint-Cloud propose à l'internaute un lexique (français) de cancérologie (<http://www.arcs.asso.fr/content/lexique.htm $>$ ). De même, la Heymans Institute of Pharmacology de l'Université de Gent et le département de linguistique appliquée de la Mercator School ont élaboré un glossaire multilingue de termes médicaux (<http://allserv.rug.ac.be/ rvdstich/eugloss/ welcome.html $>$ ) qu'il est possible de consulter en ligne. Les sites d'organisations du secteur de la santé représentent donc des sources possibles d'ouvrages terminologiques ou, mieux, d'inventaires d'ouvrages terminologiques. L'Organisation mondiale de la santé a notamment dressé une liste d'ouvrages de référence en médecine $(<$ http://www.who.int/terminology/ter/dicfair.html $>)$, liste qui regroupe une cinquantaine d'ouvrages classés par domaines de spécialisation. Les répertoires spécialisés en sciences de la santé, dont il a été question à la section 1, constituent également une autre piste à explorer pour localiser des ressources dictionnairiques en ligne. En effet, il n'est pas rare qu'ils donnent accès à des listes de dictionnaires ou de lexiques.

Certains organismes reconnus en matière de langue ou de terminologie ont aussi établi des listes de répertoires terminologiques pouvant être consultés sur Internet. L'Office de la langue française (<http://www.olf.gouv.qc.ca $>)$ diffuse sur son site un Inventaire des terminologies dans Internet. En juillet 2000, l'inventaire regroupait plus de cent liens vers des ouvrages du domaine des sciences de la santé accessibles en ligne, pour la plupart des lexiques bilingues ou unilingues français élaborés par divers organismes ou groupes de recherche. Il s'agit là de l'inventaire le plus complet à ce jour en matière de ressources terminologiques médicales de langue française. De même, la Délégation générale à la langue française $(<\mathrm{http}: / /$ www. culture.fr/culture/dglf/garde.htm $>$ ) a constitué une liste de ressources terminologiques électroniques dans laquelle figurent quelques dictionnaires médicaux.

Enfin, il existe des répertoires de dictionnaires qui, sans être consacrés essentiellement au domaine médical, donnent néanmoins accès à des ouvrages de ce domaine. On peut penser au répertoire OneLook Dictionaries ( $<$ http://www.onelook.com/ browse.shtml\#all_med $>$ ), qui contient une cinquantaine de liens vers des ressources terminologiques en médecine. De même, on compte des dictionnaires médicaux parmi les dictionnaires, encyclopédies et glossaires électroniques recensés par le Service des bibliothèques de l'Université de Sherbrooke (<http://www.biblio.usherb. $\mathrm{ca} /$ internet/dictext.htm $>$ ). Une liste de répertoires de dictionnaires est proposée en annexe.

Pour conclure sur la question des ressources terminologiques en ligne, il faut garder à l'esprit que les dictionnaires, lexiques et glossaires accessibles sur Internet ne sont pas tous élaborés par des terminologues et que, en conséquence, ils ne respec- 
tent pas nécessairement les règles de l'art. Il est donc préférable de valider l'information qui s'y trouve dans d'autres sources, électroniques ou traditionnelles. Mais les ouvrages dictionnairiques en ligne n'en demeurent pas moins, dans bien des cas, d'excellentes pistes pour résoudre des problèmes terminologiques et favoriser la compréhension de certaines notions.

\section{Conclusion}

Il ne fait pas de doute qu'Internet constitue une ressource documentaire exploitable par le traducteur médical. Ce dernier peut en effet y consulter un nombre inestimable de documents de toute nature liés à son domaine de spécialité. Toutefois, pour s'assurer de la validité de l'information repérée, il devra évaluer la qualité générale du contenu du site d'où provient cette information. Différents outils d'évaluation de sites Web ont été développés pour venir en aide à tous ceux qui comptent utiliser les ressources d'Internet à des fins professionnelles. Le Réseau canadien de la santé a notamment mis au point une liste de vérification $(<\mathrm{http}$ ://www.reseau-canadiensante.ca/html/helpf/netinfof.html $>$ ) qui prend la forme d'une série de questions que l'usager devrait se poser lorsqu'il consulte un site Web. La British Library et l'Université d'Oxford proposent également un instrument d'évaluation intéressant, le Discern Instrument (<http://www.discern.org.uk/discern__instrument.htm $>)$. FrançoisPierre Gingras, professeur à l'Université d'Ottawa, a pour sa part préparé un document qui s'intitule L'utilisation des ressources d'Internet et les travaux scientifiques: questions d'appréciation (<http://aix1.uottawa.ca/ fgingras/text/sources.html >); l'auteur attire l'attention sur un certain nombre d'éléments qui devraient être pris en considération pour juger de la valeur de l'information diffusée sur le Web.

\section{NOTES}

1. On les appelle également catalogues, index ou simplement listes d'adresses.

2. Les ressources en langue anglaise sont nettement prédominantes sur le Web. Toutefois, cette tendance générale semble moins marquée en sciences de la santé que dans d'autres secteurs d'activités. Le traducteur médical qui traduit vers le français pourra donc trouver sur Internet une somme importante de ressources documentaires en langue française.

\section{RÉFÉRENCES}

BADOR, P. (s. d.): «Les sources d'information pharmaceutique sur Internet», Unité régionale de formation et de promotion pour l'information scientifique et technique, <http://urfist.univlyon 1.fr/pharmacie.html> (28 juin 2000).

Delisle, J. (1997): La traduction raisonnée. Manuel d'initiation à la traduction professionnelle anglais $\varnothing$ français, Ottawa, Presses de l'Université d'Ottawa, coll. «Pédagogie de la traduction».

Didier, B. (2000): «La recherche d'information biomédicale sur Internet», Bibliothèque de l'Institut Pasteur, <http://www.pasteur.fr/infosci/biblio/ribi.html> (28 juin 2000).

Éveillard, P. (2000): "Suicide collectif sur la Toile médicale française», La revue du praticien, 14-488, p. 327-328.

Jammal, A. (1999) : «Une méthodologie de la traduction médicale», Meta, 44-1, p. 217-237.

LARDY, J.-P. (s. d.) : "La recherche d'informations sur différents services», Unité régionale de formation et de promotion pour l'information scientifique et technique, <http://urfist.univ-lyon1.fr/ recherch.html> (25 juin 2000). 
140 Meta, XLVI, 1, 2001

ANNEXE

1. Répertoires spécialisés en sciences de la santé

R

Bibliothèque médicale $\mathrm{A}$. F. Lemanissier

Bibliothèque médicale A. F. Lemanissier

$<$ http://www.cybercable.tm.fr/ biblioa $>$

Centre hospitalier universitaire de Rouen

Catalogue et index des sites médicaux francophones (CISMeF)

$<$ http://www.chu-rouen.fr/cismef $>$

Promedical.net

Promedical.net

$<$ http://www.promedical.net>

Société de services et de conseils en informatique médicale et télématique

Medintel

$<$ http://www.medintel.net/fr/index2.htm $>$

$R$

Centre d'information sur la santé de l'enfant, Hôpital Sainte-Justine Sélection des meilleurs sites en santé

$<$ http://brise.ere.umontreal.ca/ lecomptl/postmenu.htm>

Association médicale canadienne

Liens WebMed

$<$ http://www.cma.ca/webmed-f $>$

Institut canadien de l'information scientifique et technique (ICIST)

Sites branchés en sciences biologiques et sciences médicales

$<$ http://www.nrc.ca/zone/cisti/special/hotlinks/biolof.shtml>

Hôpital Maisonneuve-Rosemont, Bibliothèque médicale

Hyperliens

$<$ http://brise.ere.umontreal.ca/ hinseo/hyperliens.html>

Université de Genève, Faculté de médecine

Adresses utiles

$<$ http://www.medecine.unige.ch/webetu/cours/adr_int.htm $>$

Université de Lausanne, Faculté de médecine

Bibliothèque et centre de documentation de la Faculté de médecine

$<$ http://www.hospvd.ch/public/chuv/bdfm>

Université de Montréal, Bibliothèque des sciences de la santé

Bibliothèque des sciences de la santé

$<$ http://www.bib.umontreal.ca/SA>

Université d'Ottawa, Réseau des bibliothèques

Ressources biomédicales sur Internet

$<$ http://www.uottawa.ca/library/health/internef.html>

\section{R}

American Medical Informatics Association's Internet Working Group Medical Matrix

$<$ http://www.medmatrix.org/reg/login.asp $>$ 
Canadian Society for Pharmaceutical Sciences/Société canadienne des sciences pharmaceutiques Global Links

$<$ http://www.ualberta.ca/ csps/links.htm>

Chungbuk National University, Medical Resource Information Center

MedMark

$<$ http://www.medmark.org >

Dalhousie University, College of Pharmacy

Useful Links

$<$ http://www.dal.ca/ pharmwww/links>

Emory University, Robert W. Woodruff Health Sciences Center Library

MedWeb

$<$ http://WWW.MedWeb.Emory.Edu/MedWeb $>$

Harvard University, Countway Library of Medicine

Countway Library of Medicine

$<$ http://countweb.med.harvard.edu>

Health Web

Health Web

$<$ http://healthweb.org >

Karolinska Institutet (Suède)

Resources

$<$ http://www.kib.ki.se/index_en.html>

McGill University, Health Sciences and Osler Libraries

Health Sciences Resources on the Internet

$<$ http://www.health.library.mcgill.ca/resource/page.htm>

McMaster University, Health Sciences Library

Guides to Internet Resources

$<$ http://www-hsl.mcmaster.ca/guides.html>

Local and Canadian Health-Related Web Sites

$<$ http://www-hsl.mcmaster.ca/links.html>

Searching for Health Sciences Resources on the Internet

$<$ http://www-hsl.mcmaster.ca/dirs.html >

National Institutes of Health, National Library of Medicine

MedlinePlus

$<$ http://medlineplus.gov $>$

National Network of Libraries of Medicine

Biosites

$<$ http://www.library.ucsf.edu/biosites $>$

Université libre de Bruxelles, Bibliothèque centrale de médecine

Bibliothèque centrale de médecine

$<$ http://www.bib.ulb.ac.be/Bcm>

University of Oklahoma, College of Pharmacy

Virtual Library Pharmacy

$<$ http://www.cpb.uokhsc.edu/pharmacy>

University of Iowa, Hardin Library for the Health Sciences

Hardin Meta Directory of Internet Health Sources

$<$ http://www.lib.uiowa.edu/hardin/md $>$ 
Yale University, Cushing/Whitney Medical Library Selected Internet Resources

$<$ http://www.med.yale.edu/library/sir>

\section{Répertoires spécialisés en sciences}

$R$

Institut national des sciences appliquées de Lyon Sapristi

$<$ http://csidoc.insa-lyon.fr/sapristi/digest.html>

R

Academic Info, catégorie «Health \& Medicine» $<$ http://www.academicinfo.net/med.html $>$

Argus Clearinghouse

$<$ http://www.clearinghouse.net>

Infomine

$<$ http://infomine.ucr.edu/search/bioagsearch.phtml $>$

\section{Portails médicaux}

$R$

Atmedica

$<$ http://www.atmedica.com>

Caducée.net

$<$ http://www.caducee.net $>$

CSanté.com

$<$ http://www.comsante.com>

Egora

$<$ http://www.egora.fr>

Galaxie Médecine

$<$ http://medecine.galaxie.fr>

Medisite

$<$ http://www.medisite.fr>

Planet Medica

$<$ http://www.planetmedica.fr $>$

Réseau Proteus (médecines alternatives)

$<$ http://www.reseauproteus.net $>$

Santé-Net Québec

$<$ http://www.pageweb.qc.ca/sante/default.htm>

$R$

Achoo

$<$ http://www.achoo.com >

C-Health

$<$ http://www.canoe.ca/Health/home.html> 
Health A to $Z$

$<$ http://www.healthatoz.com/atoz/searchbycat.asp $>$

Health Oasis - Mayo Clinic

$<$ http://www.mayohealth.org >

InteliHealth

$<$ http://www.intelihealth.com>

MedExplorer

$<$ http://www.medexplorer.com>

Medscape

$<$ http://www.medscape.com $>$

OnHealth

$<$ http://www.onhealth.com>

\section{Répertoires et moteurs de recherche généraux}

R

Nomade

$<$ http://www.nomade.fr $>$

La Toile du Québec

$<$ http://www.toile.qc.ca>

Yahoo France

$<$ http://fr.dir.yahoo.com/Sante>

R

About.com

$<$ http://www.about.com>

AltaVista

$<$ http://www.altavista.com>

Google

$<$ http://www.google.com>

HotBot

$<$ http://www.hotbot.com>

Infoseek

$<$ http://www.infoseek.com>

Northern Light

$<$ http://www.northernlight.com>

Yahoo Canada

$<$ http://ca.yahoo.com/Health>

\section{Inventaires de ressources terminologiques}

Centre d'information sur la santé de l'enfant

Encyclopédies et dictionnaires en ligne

$<\mathrm{http}$ ///brise.ere.umontreal.ca/ lecomptl/postmen2.htm\#405>

ClicNet

Dictionnaires et lexiques

$<$ http://clicnet.swarthmore.edu/dictionnaires.html $>$ 
144 Meta, XLVI, 1, 2001

Délégation générale à la langue française

De la terminologie en ligne

$<$ http://www.culture.fr/culture/dglf/garde.htm>

Jim Martindale

Medical Dictionaries

$<$ http://www-sci.lib.uci.edu/HSG/Medical.html\#DICTION>

Medline Plus

Dictionaries

<http://www.nlm.nih.gov/medlineplus/dictionaries.html>

Michigan Electronic Library

Reference Desk

$<$ http://mel.lib.mi.us/reference/REF-dict.html>

Office de la langue française

Inventaire des terminologies sur Internet

$<$ http://www.olf.gouv.qc.ca $>$

Science.komm

Online Scientific and Medical Dictionaries

$<$ http://www.sciencekomm.at/advice/dict.html\#med>

Study Technologies

OneLook Dictionaries

$<$ http://www.onelook.com/browse.shtml>

StudyWeb

Reference

<http://www.studyweb.com/links/675.html>

Université de Sherbrooke, Service des bibliothèques

Dictionnaires, encyclopédies et glossaires

$<$ http://www.biblio.usherb.ca/internet/dictext.htm>

yourDictionary.com

A Web of On-line Dictionaries

$<$ http://www.yourdictionary.com> 